\title{
STRUCTURE THEORY OF FAITHFUL RINGS, III. IRREDUCIBLE RINGS
}

\author{
R. E. JOHNSON
}

The first two papers of this series ${ }^{1}$ were primarily concerned with a closure operation on the lattice of right ideals of a ring and the resulting direct-sum representation of the ring in case the closure operation was atomic. These results generalize the classical structure theory of semisimple rings. The present paper studies the irreducible components encountered in the direct-sum representation of a ring in (F II). For semisimple rings, these components are primitive rings. Thus, primitive rings and also prime rings are special instances of the irreducible rings discussed in this paper.

1. Introduction. Let $L_{r}(R)$ and $L_{l}(R)$ designate the lattices of $r$-ideals and $l$-ideals, respectively, of a ring $R$. If $M$ is an $(S, R)$ module, $L_{r}(M)$ designates the lattice of $R$-submodules of $M$, and similarly for $L_{l}(M)$. For every lattice $L$, we let $L^{\boldsymbol{\Delta}}=\{A \mid A \in L$, $A \cap B \neq 0$ for every nonzero $B \in L\}$. The elements of $L^{\wedge}$ are referred to as the large elements of $L$.

If $M$ is an $(S, R)$-module and $A$ and $B$ are subsets of $M$, then let $A B^{-1}=\{s \mid s \in S, s B \subset A\}$ and $B^{-1} A=\{r \mid r \in R, B r \subset A\}$. In particular, if $x \in M$ then $x^{-1} 0\left(0 x^{-1}\right)$ is the right (left) annihilator of $x$ in $R(S)$. The set $M_{r}^{\mathbf{\Delta}}=\left\{x \mid x \in M, x^{-1} 0 \in L_{r}^{\mathbf{\Delta}}(R)\right\}$ is an $(S, R)$-submodule of $M$ called the right singular submodule. If we consider $R$ as an $(R, R)$-module, then $R_{r}^{\mathbf{A}}$ is an ideal of $R$ called the right singular ideal in [6]. It is clear how $M_{l}^{\mathbf{\Lambda}}$ and $R_{l}^{\mathbf{\Lambda}}$ are defined and named.

A primitive ring with minimal $r$-ideals is an example of a ring having a zero singular ideal. An example to be given later, the ring of all $n \times n$ triangular matrices over $Z$, the ring of integers, shows that a ring can have both a zero singular ideal and a nonzero radical.

It is useful at times to extend a ring $R$ without unity to one with unity before discussing its properties. We may let

$$
R_{1}=\{(r, n) \mid r \in R, n \in Z\}
$$

be the usual extension of $R$ to a ring with unity (operations being defined in $R_{1}$ as if $\left.(r, n)=r+n\right)$, then $R^{\prime}=\{(r, 0) \mid r \in R\}$ is an ideal of

Received by the editors December 3, 1959.

1 I. Closureoperations on lattices; II. Restricted rings, Trans. Amer. Math. Soc. vol. 84 (1957) pp. 508-522, 523-544. These papers are referred to as (F I) and (F II), respectively. The first fifteen items of the bibliography are at the end of (F I); more recent items are listed at the end of this paper. 
$R_{1}$ isomorphic to $R$. If we let $R_{0}$ be a maximal element in the set of all ideals of $R_{1}$ having zero intersection with $R^{\prime}$, then the factor ring $R_{1} / R_{0}$ has a unity and contains (an isomorphic image of) $R$. If either $0 R^{-1}=0$ or $R^{-1} 0=0$, then the ideal $R_{0}$ is unique; it is either the left or the right annihilator of $R^{\prime}$ in $R_{1}$. In case $R_{0}$ is unique, so is the factor ring $R_{1} / R_{0}$. This unique extension of $R$ to a ring with unity will be designated by $R_{u}$. Evidently $R$ is a large ideal of $R_{u}, L_{r}(R)$ $\subset L_{r}\left(R_{u}\right)$ and similarly for $l$-ideals.

We recall that if $A, B \in L$, a lattice, then $B$ is called an essential extension of $A$, and we write $A C^{\prime} B$, if $B \supset A$ and if $C \cap A \neq 0$ whenever $C \cap B \neq 0, C \in L$. If $M$ is a right $R$-module such that $M_{r}^{\mathbf{\Delta}}=0$, then it is shown in (F II, §6) that each $A \in L_{r}(M)$ has a unique maximal essential extension $A^{s} \in L_{r}(M)$. The mapping $s: A \rightarrow A^{s}$ is a closure operation on $L_{r}(M)$ with properties as stated in (F II, §6). We designate the lattice of closed submodules of $M$ by $L_{r}^{s}(M)$.

The proof of the first part of the following theorem is straightforward and is therefore omitted. The second part follows from (F II, $6.8)$.

1.1. Theorem. If $R$ is a ring such that $R_{r}^{\Delta}=0$, then, considering $R$ and $R_{u}$ as right $R$-modules, $R C^{\prime} R_{u}$ and $R_{u r}^{\mathbf{\Lambda}}=0$. Also, $L_{r}^{s}\left(R_{u}\right) \cong L_{r}^{s}(R)$ under the correspondence $A \leftrightarrow A \cap R, A \in L_{r}^{s}\left(R_{u}\right)$.

Each right $R$-module $M$ becomes a unitary $R_{1}$-module when we define

$$
x(a, n)=x a+n x, \quad x \in M, \quad(a, n) \in R_{1} .
$$

(See (F II, p. 542), $[16 ; 17])$. It is unitary because $(0,1)$ is the unity element of $R_{1}$ and $x(0,1)=x$. Since $R_{0} R=0,\left(x R_{0}\right) R=0$ and therefore $x R_{0}=0$ for every $x \in M$ if $M_{r}^{\boldsymbol{\Lambda}}=0$. Thus, if $M_{r}^{\boldsymbol{\Lambda}}=0, M$ becomes a unitary $R_{u}$-module if we define

$$
x\left[(a, n)+R_{0}\right]=x a+n x, \quad x \in M, \quad(a, n)+R_{0} \in R_{u} .
$$

If $M_{r}^{\boldsymbol{\Delta}}=0$ considering $M$ as an $R$-module, then also $M_{r}^{\boldsymbol{\Delta}}=0$ considering $M$ as an $R_{u}$-module. It is true, conversely, that if $M$ is a right $R_{u}$-module such that $M_{r}^{\mathbf{\Delta}}=0$, then as a right $R$-module also $M_{r}^{\mathbf{\Delta}}=0$. If $M$ and $N$ are right $R$-modules such that $M_{r}^{\mathbf{\Delta}}=N_{r}^{\boldsymbol{\Delta}}=0$ and $M \subset N$, then $M C^{\prime} N$ as right $R$-modules if and only if $M C^{\prime} N$ as right $R_{u}$ modules.

An $R$-module $M$ is injective if for every $R$-module $N$ and every extension module $N^{\prime}$ of $N$, each homomorphism of $N$ into $M$ can be extended to one of $N^{\prime}$ into $M$. In order to show that a module $M$ is injective, it suffices to show that for each essential extension $N^{\prime}$ of $N$ 
every homomorphism of $N$ into $M$ can be extended to one of $N^{\prime}$ into $M$. It is an important result of ring theory that every $R$-module $M$ has a unique minimal injective extension $\hat{M} .^{2}$

A simple realization of $\hat{M}$ in case $M_{r}^{\boldsymbol{\Delta}}=0$ is as follows:

$$
\hat{M}=\bigcup_{A \in L_{\boldsymbol{r}}(R)} \operatorname{Hom}_{R}(A, M) .
$$

For $x, y \in \hat{M}$, we take $x=y$ if and only if $x a=y a$ for every $a \in A, A$ some large $r$-ideal of $R$. The domain of $x+y$ is the intersection of the domains of $x$ and $y$; that of $x r$ is $r^{-1} A$ where $A$ is the domain of $x$. (See (F II, top of p. 543); [18] and [24].)

It is easily shown that if $R$ is a ring without unity and if $M$ is an $R$-module such that $M_{r}^{\boldsymbol{\Lambda}}=0$, then the minimal injective extension of $M$ as an $R$-module coincides with that of $M$ considered as an $R_{u}$ module.

2. Irreducible rings. A ring $R$ will be called (right) irreducible if $R_{r}^{\mathbf{\Lambda}}=0$ and if there exists no nonzero ideal $S$ of $R$ such that $0 S^{-1} \neq 0$ and $S \cap 0 S^{-1}=0$. In the language of (F II, p. 524), this latter condition is equivalent to $F^{\prime \prime}(R)=\{0, R\}$ since $F(R)=F^{\prime}(R)$ if $R_{r}^{\mathbf{\Lambda}}=0$ (F II, p. 538). The lattice $L_{r}^{s}(R)$ is simple for an irreducible ring by (F II, 6.7).

A nonzero right $R$-module $M$ will be called irreducible if $M_{r}^{\Delta}=0$ and $A \cap B \neq 0$ for every nonzero $A, B \in L_{r}(M)$. This latter condition is equivalent to either $L_{r}^{\mathbf{\Delta}}(M)=L_{r}(M)-\{0\}$ or $L_{r}^{s}(M)=\{0, M\}$. If $L_{r}^{s}(R)$ is atomic, then each atom of $L_{r}^{s}(R)$ is an irreducible right $R$ module.

If $L_{r}^{s}(R)$ is atomic and if every nonzero $A \in L_{r}^{s}(R)$ contains an atom, then the closure operation $s$ is called homogeneous (F I, p. 513). If $s$ is homogeneous on $L_{r}(R)$ and if $M$ is a right $R$-module such that $M_{r}^{\boldsymbol{\Delta}}=0$, then $s$ also is homogeneous on $L_{r}(M)$. By (F II, 6.8), $(x R)^{s}$ is an atom of $L_{r}^{s}(M)$ if and only if $x^{-1} 0$ is a maximal element $(\neq R)$ if $L_{r}^{s}(R)$. Consequently, if $M$ is irreducible then $x^{-1} 0$ is a maximal element of $L_{r}^{s}(R)$ for every nonzero $x \in M$.

Two atoms $A$ and $B$ of $L_{r}^{s}(R)$ or $L_{r}^{s}(M)$ are called perspective if and only if $a^{-1} 0=b^{-1} 0$ for some nonzero $a \in A$ and $b \in B$. If $R$ is irreducible, every pair of atoms of $L_{r}^{s}(R)$ is a perspective pair by remarks following (F II, 6.12). The ring union of the atoms of $L_{\tau}^{s}(R)$ is an ideal of $R$ called the base of $R$ and designated by $R_{r}^{*}$. If $s$ is homogeneous, then clearly $R_{r}^{*} \in L_{r}^{\boldsymbol{\Lambda}}(R)$.

2 For rings with unity, this result is due to Baer [1]. An elegant proof of it may be found in [4]. The trivial extension of this result to rings without unity may be found in (F II, p. 542). 
We remark in passing it may be shown that if $R$ is an irreducible ring then either $R$ has prime characteristic or every nonzero element of $R$ has characteristic zero.

If $A$ and $B$ are nonzero ideals of a prime ring $R$, then $A \cap B \neq 0$. Thus, a prime ring $R$ for which $R_{r}^{\mathbf{\Lambda}}=0$ is an example of an irreducible ring. The ring $R$ of all $n \times n$ triangular matrices over $Z$ is irreducible, as we shall show in the next section. This ring clearly is not prime, since it has nilpotent ideals.

2.1. Theorem. An irreducible ring is prime if and only if $R$ contains no nonzero nilpotent ideals.

Proof. If the ring $R$ is not prime, then $R$ contains nonzero ideals $A$ and $B$ such that $A B=0$. If $A \cap B \neq 0$, then $R$ contains a nilpotent ideal, namely $A \cap B$. Otherwise, if $C \cap D=0$ for every pair of ideals such that $C D=0$, then not only is $A \cap B=0$ but also $A \cap 0 A^{-1}=0$. This, $R$ is not irreducible.

If the ring $R$ is irreducible and the closure operation $s$ on $L_{r}(R)$ is homogeneous, and if $M_{1}$ and $M_{2}$ are irreducible right $R$-modules, then there exist nonzero $x_{i} \in M_{i}$ such that $x_{1}^{-1} 0=x_{2}^{-1} 0$. This is so since for any nonzero $y_{i} \in M_{i}$, there exist atoms $A_{i} \in L_{r}^{s}(R)$ such that $y_{i} A_{i} \neq 0, i=1,2$. Thus, $A_{1}$ and $A_{2}$ are perspective and $a_{1}^{-1} 0=a_{2}^{-1} 0$ for some nonzero $a_{i} \in A_{i}$. The desired conclusion follows with $x_{i}=y_{i} a_{i}$. The minimal injective extension of an irreducible $R$-module is irreducible by (F II, 6.8).

2.2. Theorem. An irreducible ring $R$ for which $s$ is homogeneous has a unique irreducible injective module $M$. Furthermore, $M$ is faithful.

Proof. Let $M_{1}$ and $M_{2}$ be irreducible injective $R$-modules. There exist nonzero $x_{i} \in M_{i}$ such that $x_{1}^{-1} 0=x_{2}^{-1} 0$. The mapping $\alpha$ of $x_{1} R$ into $M_{2}$ defined by $\alpha\left(x_{1} r\right)=x_{2} r, r \in R$, is a homomorphism. As such, it can be extended to a homomorphism $\alpha^{\prime}$ of $M_{1}$ into $M_{2}$. For each nonzero $x \in M_{1}, x a=x_{1} b \neq 0$ for some $a, b \in R$. Since $\alpha^{\prime}(x a)=\alpha\left(x_{1} b\right)$ $=x_{2} b \neq 0$, evidently $\alpha^{\prime} x \neq 0$. Thus, $\alpha^{\prime}$ is an isomorphism of $M_{1}$ into $M_{2}$. Since $\alpha^{\prime} M_{1}$ is injective and $\alpha^{\prime} M_{1} \subset^{\prime} M_{2}$, and since an injective module has no essential extension, evidently $\alpha^{\prime} M_{1}=M_{2}$. Hence $M_{1} \cong M_{2}$ and $R$ has a unique (up to isomorphism) irreducible injective module $M$.

We shall prove that $M$ is faithful by transferring our argument to the ring $R_{u}$. Each atom $A \in L_{r}^{s}(R)$ is contained in a unique atom $A^{\prime} \in L_{r}^{s}\left(R_{u}\right)$. Since $M$ is also an injective $R_{u}$-module, there exists an isomorphic mapping $\beta$ of the right $R_{u}$-module $A^{\prime}$ into $M$ by an argument similar to that of the preceding paragraph. Since $M$ is injective, $\beta$ can be extended to a homomorphism $\beta^{\prime}$ of $R_{u}$ (as a right $R_{u}$ - 
module) into $M$. If $\beta^{\prime} 1=x$, then $\beta a=x a$ for every $a \in A^{\prime}$. Therefore, $x A \neq 0$, and we conclude that $M A \neq 0$ for every atom $A \in L_{r}^{8}(R)$. Hence $M$ is faithful.

The ring $\operatorname{Hom}_{R}(M, M)$ associated with this irreducible injective module $M$ is a division ring (F II, 7.5). Consequently, we have proved that each irreducible ring $R$ for which $s$ is homogeneous is a ring of endomorphisms of a vector space over some division ring.

It need hardly be remarked that the concepts discussed above apply equally to the left structure of a ring.

3. Irreducible rings of endomorphisms. If $M$ is an abelian group, then a subring $R$ of $\operatorname{Hom}(M, M)$ will be called an irreducible ring of endomorphisms of $M$ if

3.1. $x R \cap y R \neq 0$ for every nonzero $x, y \in M$, and

3.2. $M_{r}^{\Lambda}=0$, considering $M$ as a right $R$-module.

Each irreducible ring for which the closure operation $s$ is homogeneous is an irreducible ring of endomorphisms by results of the preceding section.

Let us show that the ring $R$ of $n \times n$ triangular matrices over $Z$ is an irreducible ring of endomorphisms of some abelian group $M$. If we let $M=Z x_{1}+Z x_{2}+\cdots+Z x_{n}$, a direct sum of $n$ copies of the additive group of $Z$, then $R$ may be interpreted as follows:

$$
R=\left\{r \mid r \in \operatorname{Hom}(M, M), x_{i} r \in \sum_{j=1}^{i} Z x_{j}\right\} .
$$

If $x=\sum m_{i} x_{i}$ and $y=\sum n_{i} x_{i}$, with $m_{j} \neq 0, n_{k} \neq 0$ and $j \leqq k$, then select $r, r^{\prime} \in R$ as follows: $x_{i} r=\delta_{i j} n_{k} x_{j}, x_{i} r^{\prime}=\delta_{i k} m_{j} x_{j}, i=1, \cdots, n$. Evidently $x r=m_{j} n_{k} x_{j}=y r^{\prime}$ so that 3.1 is satisfied. If we let $e_{j}$ be the idempotent defined by $x_{i} e_{j}=\delta_{i j} x_{i}, i=1, \cdots, n$, then $x e_{j}=m_{j} x_{j} \neq 0$. It is clear that $x^{-1} 0 \cap e_{j} R=0$, since $x_{j} r=0$ implies $x_{j} e_{j} r=0$ and $e_{j} r=0$. Thus 3.2 is also satisfied by $R$.

If $R$ is an irreducible ring of endomorphisms of an abelian group $M$ and if $r \in R, r \neq 0$, then $x r \neq 0$ for some $x \in M$. By 3.2, there exists a nonzero $A \in L_{r}(R)$ such that $(x r)^{-1} 0 \cap A=0$. Hence $r^{-1} 0 \cap A=0$, and we conclude that $R_{r}^{\mathbf{\Lambda}}=0$. Therefore the closure operation $s$ is defined both on $L_{r}(R)$ and $L_{r}(M)$.

3.4. THEOREM. If $R$ is an irreducible ring of endomorphisms of an abelian group $M$, then the closure operation son $L_{r}(R)$ is homogeneous.

Proof. For each nonzero $x \in M$, we select $A \in L_{r}(R)$ so that $x^{-1} 0 \cap A=0$ and $x^{-1} 0 \cap B \neq 0$ if $B \supset A, B \neq A$. Since $\left(x^{-1} 0 \cap A\right)^{s}$ $=x^{-1} 0 \cap A^{s}=0$, evidently $A^{s}=A$ and $A \in L_{r}^{s}(R)$. Let us prove that $A$ 
is an atom. If there exists some nonzero $B \in L_{r}^{s}(R)$ such that $B \subset A$ and $B \neq A$, then there also exists some $C \in L_{r}^{s}(R)$ such that $C \cap B=0$ and $C \cap A \neq 0$ (F I, p. 520). If we let $D=C \cap A$, then $B+D \subset A$, $B \cap D=0$. Since $x B \neq 0$ and $x D \neq 0$, also $x B \cap x D \neq 0$ by 3.1. If $x b=x d$ $\neq 0$ for $b \in B, d \in D$, then also $x(b-d)=0$ and $b-d \in x^{-1} 0 \cap A$. Thus $b-d=0$ and $b=d$. This implies that $B \cap D \neq 0$ contrary to the choice of $D$. Hence $A$ must be an atom of $L_{r}^{s}(R)$.

If $B \in L_{r}^{s}(R), B \neq 0$, then $x B \neq 0$ for some $x \in M$. Since $x^{-1} 0 \cap B \neq B$ and $x^{-1} 0 \cap B \in L_{r}^{s}(R)$, there exists some nonzero $A \in L_{r}^{s}(R)$ such that $A \subset B$ and $A \cap x^{-1} 0=0$. Thus $B$ contains an atom $A$ and $s$ is homogeneous.

If $A$ and $B$ are atoms of $L_{r}^{s}(R)$ and $x, y \in M$ are chosen so that $x A \neq 0$ and $y B \neq 0$, then $x A \cap y B \neq 0$ by 3.1. Hence $x a=y b \neq 0$ for some $a \in A, b \in B$, and $(x a)^{-1} 0=(y b)^{-1} 0=a^{-1} 0=b^{-1} 0$. Thus the atoms $A$ and $B$ are perspective, and $R$ is irreducible by (F II, 6.12). This proves the following result.

3.5. ThEOREM. Each irreducible ring of endomorphisms of an abelian group is an irreducible ring for which the closure operation $s$ is homogeneous.

We may now conclude, for example, that the ring $R$ (of 3.3) of all $n \times n$ triangular matrices over $Z$ is irreducible. Furthermore, its closure operation $s$ is homogeneous.

4. Primitive rings. If $R$ is a ring such that $R_{r}^{\Delta}=0$ and if $e$ is an idempotent of $R$, then $e R \in L_{r}^{s}(R)$. This is so since $e R \cap(1-e) R=0$ whereas $A \cap(1-e) R \neq 0$ for any $A \supset e R, A \neq e R$. Hence, if $A$ is a minimal $r$-ideal of $R$ for which $A^{2} \neq 0$, then $A \in L_{r}^{s}(R)$ since $A=e R$ for some idempotent $e \in R$.

Each ring $R$ considered in this section is assumed to be primitive and to contain minimal $r$-ideals. It is easily seen that $R_{r}^{\mathbf{A}}=0, s$ is homogeneous and the atoms of $L_{r}^{s}(R)$ are the minimal $r$-ideals for such a ring $R$. Thus, the base $R_{r}^{*}$ of $R$ is its right socle [20, p. 64]. The right and left socles are equal for a primitive ring, so the right and left bases must be equal. Every large $r$-ideal of $R$ must contain every minimal $r$-ideal, and therefore must contain the base $R^{*}$.

The ring $R$ has a unique faithful simple right $R$-module $M$, which we may assume to have the form $M=e R, e R$ an atom of $L_{r}^{s}(R)$ with idempotent generator $e[20$, p. 64 et. seq.]. The $l$-ideal $R e$ also is minimal, and $e R e$ is a division ring isomorphic to $\operatorname{Hom}_{R}(M, M)$.

Since $R^{*}$ is contained in each large $r$-ideal, our previous characterization of $\hat{M}$ has the simple form 


$$
\hat{M}=\operatorname{Hom}_{R}\left(R^{*}, M\right)
$$

for a primitive ring with minimal $r$-ideals. Each $x \in \hat{M}$ maps the subring $R e$ of $R^{*}$ into the subgroup $e R e$ of $M$. Thus, if $x(r e)=e a$, then $[x(r e)] e=x(r e)=e a e$ and $e a=e a e$. If $x R e=0$, then $x A=0$ for every atom $A \in L_{r}^{s}(R)$ since $\operatorname{Re} A \neq 0$. Hence $x=0$, and each $x \in \hat{M}$ is uniquely determined by what it does to $R e$. That is, each $x \in \hat{M}$ is associated with a unique element of

$$
N=\operatorname{Hom}_{e R e}(R e, e R e) .
$$

It is true, conversely, that each $x \in N$ has a unique extension $x^{\prime} \in \hat{M}$. To prove this, we use the fact that $R^{*}$ is a direct sum of the atoms of $L_{r}^{s}(R), R^{*}=\sum_{i} A_{i}$, and that $\operatorname{Re} \cap A_{i} \neq 0$ for each $i$. If we select some nonzero $a_{i}$ in each $\operatorname{Re} \cap A_{i}$, then $A_{i}=a_{i} R$ and each $b \in R^{*}$ has the form $b=\sum a_{i} r_{i}, r_{i} \in R$. Let us define $x^{\prime} b$ as follows: $x^{\prime} b=\sum\left(x a_{i}\right) r_{i}$. We see that $x^{\prime}$ is a well-defined mapping of $R^{*}$ into $e R$, for if $b=\sum a_{i} t_{i}$ also, then $a_{i}\left(r_{i}-t_{i}\right)=0$ for each $i$. Hence $r_{i}-t_{i} \in(1-e) R$ and $\left(x a_{i}\right)\left(r_{i}-t_{i}\right)=0$ because $x a_{i} \in e R e$. Thus, $\left(x a_{i}\right) r_{i}=\left(x a_{i}\right) t_{i}$ for each $i$. It is clear that $x^{\prime} \in \hat{M}$, and we have proved the following theorem.

4.1. Theorem. If $e$ is an idempotent and $M=e R$ is an atom of $L_{r}^{s}(R)$, then the unique irreducible injective module $\hat{M}$ of $R$ may be described by

$$
\hat{M}=\operatorname{Hom}_{\theta R e}(R e, e R e) .
$$

If $D$ is a division ring and $M$ is a unitary left $D$-module, then $M^{*}=\operatorname{Hom}_{D}(M, D)$ is a unitary right $D$-module called the conjugate of $M\left[20\right.$, p. 66]. For convenience, we consider the elements of $M^{*}$ as right operators on $M$. A submodule $M^{\prime}$ of $M^{*}$ is called total if $x M^{\prime} \neq 0$ for each nonzero $x \in M$. If $E=\operatorname{Hom}_{D}(M, M)$ and $E^{*}$ $=\operatorname{Hom}_{D}\left(M^{*}, M^{*}\right)$, where $M$ is considered to be a $(D, E)$-module and $M^{*}$ a $\left(E^{*}, D\right)$-module, then $E \subset E^{*}$ if for $a \in E$ and $y \in M^{*}$ we define $a y$ as follows: $x(a y)=(x a) y, x \in M$.

If $M^{\prime}$ is a total subspace of $M^{*}$ and $R=\left\{a \mid a \in E, a M^{\prime} \subset M^{\prime}\right\}$, then $R$ is a primitive ring with minimal $r$-ideals $[20$, p. 75] having $M$ as its unique simple right $R$-module and $M^{\prime}$ as its unique simple left $R$ module. According to $4.1, \hat{M}=M^{\prime *}$ where $M^{\prime *}$ is the conjugate of the right $D$-module $M^{\prime}$.

For finite dimensional $D$-modules, $\hat{M}=M$ since $M, M^{\prime}$ and $M^{\prime *}$ all have the same dimension. If $M$ has infinite $D$-dimension, then so have $M^{\prime}$ and $M^{\prime *}$, and $\operatorname{dim} M^{\prime *}>\operatorname{dim} M^{\prime}\left[20\right.$, p. 68]. If $\operatorname{dim} M=\boldsymbol{\aleph}_{0}$, then $\operatorname{dim} M^{\prime} \geqq \boldsymbol{\aleph}_{0}$ so that $\operatorname{dim} M^{\prime *}>\boldsymbol{\aleph}_{0}$. Thus, if $\operatorname{dim} M=\boldsymbol{\aleph}_{0}, \hat{M} \neq M$ and $M$ cannot be injective. An easy example showing that $\hat{M}$ can 
equal $M$ if $M$ has infinite $D$-dimension (interchanging left and right) is afforded by the ring $E$ above. The left $E$-module $M^{*}$ has $M$ as the total subspace of its conjugate space associated with $E$, and therefore $\hat{M}^{*}=M^{*}$ by our previous remarks. That is, the simple left $E$-module $M^{*}$ is injective.

Added May 18, 1960. A recent paper by J. P. Jans, Projective injective modules, Pacific J. Math. vol. 9 (1959) pp. 1103-1108, contains a special case (Theorem 2.1) of our Theorem 4.1.

\section{BIBLIOGRAPHY}

16. A. Kertész, Beiträge zur Theorie der Operatormoduln, Acta Math. Acad. Sci. Hungar. vol. 8 (1957) pp. 235-257.

17. - A remark on the general theory of modules, Publ. Math. Debrecen vol. 6 (1959) pp. 86-89.

18. G. D. Findlay and J. Lambek, A generalized ring of quotients, I and II, Canad. Math. Bull. vol. 1 (1958) pp. 77-85, 155-167.

19. H. Cartan and S. Eilenberg, Homological algebra, Princeton, 1956.

20. N. Jacobson, Structure of rings, Amer. Math. Soc. Colloquium Publications vol. 37, 1956.

21. Y. Utumi, On quotient rings, Osaka Math. J. vol. 8 (1956) pp. 1-18.

22. A. W. Goldie, The structure of prime rings under ascending chain conditions, Proc. London Math. Soc. vol. 8 (1958) pp. 589-608.

23. R. E. Johnson, Rings with unique addition, Proc. Amer. Math. Soc. vol. 9 (1958) pp. 57-61.

24. E. T. Wong and R. E. Johnson, Self-injective rings, Canad. Math. Bull. vol. 2 (1959) pp. 167-173.

\section{Smith College}

